\section{Novel micromirror design with variable pull-in voltage}

$\underline{\text { Roel Beernaert }}{ }^{\mathrm{a}}$, Tomas Podprocky ${ }^{\mathrm{a}}$, Jeroen De Coster ${ }^{\mathrm{b}}$, Ann Witvrouw ${ }^{\mathrm{b}}$, Luc Haspeslagh ${ }^{\mathrm{b}}$, Aykut Avci ${ }^{\mathrm{a}}$, Jelle De Smet ${ }^{a}$, Herbert De Smet ${ }^{\mathrm{a}, \mathrm{b}}$

${ }^{a}$ Center of MicroSystems Technology, Ghent University, Zwijnaarde, B-9052, Belgium ${ }^{b}$ Interuniversity MicroElectronics Center, Leuven, B-3001, Belgium

\section{e-mail: Roel.Beernaert@elis.ugent.be}

Keywords: micromirror, multiple electrodes, variable pull-in, pull-in voltage.

\section{Abstract:}

A novel micromirror is presented having a variable pull-in voltage. This allows analog Pulse Width Modulation (PWM) so that the number of grey levels is not limited by the number of subframes or bitplanes. The levels can be chosen arbitrarily, allowing less severe speed requirements for the electronic layer below the MEMS, less image processing hardware and memory. The presented design of the mirror has two attracting electrodes and one landing electrode at either side of the mirror. One electrode is used as 'fixed' electrode, influencing the other attracting electrode's pull-in voltage. If we apply a general triangular waveform at the former electrode, the 'fixed' electrode voltage determines the duty cycle of the mirror. The mirror implements analog PWM, without needing a transistors based comparator at the CMOS level. When each 'fixed' electrode is connected to an active matrix cell, an active matrix display can be formed. The mirrors with variable pull-in voltage were fabricated using SiGe as the structural layer. The variable pull-in principle is demonstrated by measurements performed on these SiGe mirrors [1] (See Figure 2).

\section{INTRODUCTION}

One of the most successful examples of a Micro Electro-Mechanical System (MEMS) is Texas Instruments' Digital Micromirror Device (DMD) [2]. Aligning these mirrors into a rectangular array leads to a Spatial Light Modulator (SLM) that is used in projection displays. The micromirrors rotate over $+12^{\circ}$ and $-12^{\circ}$. Each mirror corresponds to a pixel on the screen, being white when reflecting light from the light source into the lens and hereby to the screen, being black when reflecting outside of the lens. The ratio of black and white states within a frame determines the grey level of the pixel. Binary weighed Pulse Width Modulation (PWM) with $\mathrm{n}$ subframes will give $(\mathrm{n}+1)$ linearly distributed grey levels. If the incoming light is filtered by a color-wheel that gives alternatively red, green and blue frames within $1 / 60 \mathrm{~s}$, a full color frame is projected on the screen.

An interesting phenomenon of electrostatic actuators such as the micromirrors that are presented in this work, is called pull-in [3]. Micromirrors are suspended in such a way that they are able to rotate between two positions. When a voltage difference is imposed between the mirror and an electrode underneath, the mirror is electrostatically attracted towards the electrode. When increasing this voltage difference, at a certain value the mirror pulls in to the most extreme point near to the attracting electrode. The corresponding voltage value is called the pull-in voltage. At this moment, the electrostatic force beats the mechanical counteraction of the mirror (for example the torque in a hinge). The mirror pulls in to the most extreme position at which a dedicated object leads to obstruction, e.g. a landing electrode. When decreasing the voltage difference, at a certain value the mirror releases. This is called the pull-out voltage.
In [4], analog PWM is applied to the DMD. The electrode voltage signal is the result of a comparison between analog input signals. This is done by a transistor circuit in the CMOS layer, so this analog PWM occurs at the electronic level.

In this article we present an active matrix display with a novel micromirror design containing 4 addressing electrodes (See Figure 1). The analog PWM occurs at the MEMS level. For convenience, we choose the two inner electrodes to get a fixed voltage value and the two outer electrodes, two anti-phase triangular waveforms, common for the whole matrix. We will also explain how the de-gamma operation can be implemented without sacrificing grey-level bit depth.

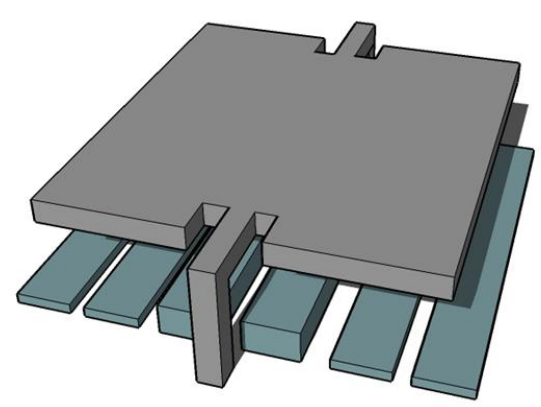

Figure 1. Micromirror design with four active electrodes and two landing electrodes.

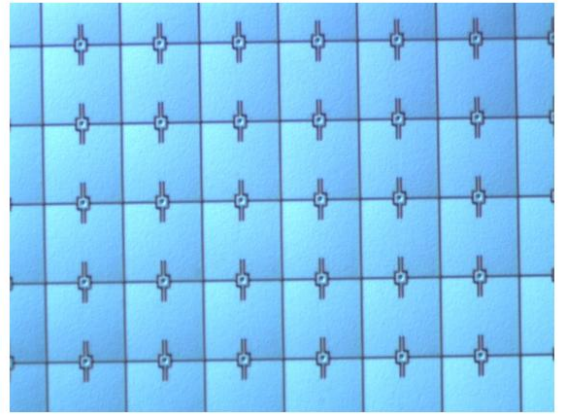

Figure 2. Top view of fabricated micromirrors using $\mathrm{SiGe}$ as the structural layer [1].

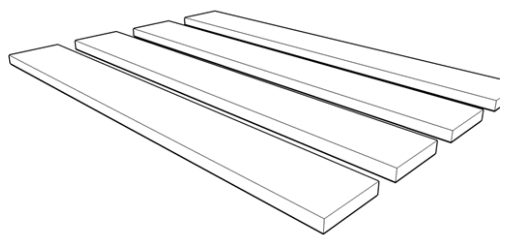

Figure 3. Configuration with four equal electrodes.

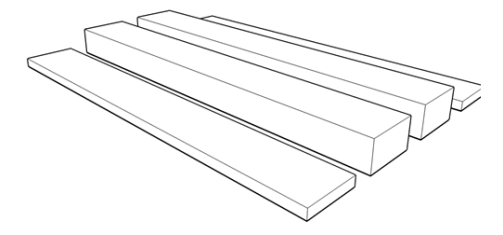

Figure 4. Configuration with two inner electrodes thicker than the two outer ones.
The simplest choice of electrodes is using four rectangular ones as in Figure 3. The inner electrode of two electrodes at a side of the mirror will have a fixed voltage value, the outer at the same side a triangular waveform. We can define the pull-in voltage of this structure as the voltage value on the outer "triangular waveform" electrode for which the mirror pulls in. By choosing the inner "fixed voltage" electrodes 
slightly elevated with respect to the outer "triangular waveform" electrodes, the former yield a stronger attraction to the mirror (closer to the mirror, see Figure 4). Our expectation is that the fixed voltage value on the central electrode can influence the pull-in voltage of this structure. This way an active matrix circuit (see Figure 5) can be used for applying and storing the fixed voltage values, as the two triangular waveforms are common for all the mirrors. The 2 inner "fixed voltage" electrodes can each have a MOSFET switch that connects their column busbar (source) to a storage capacitor (drain) if the corresponding row (gate) is high. So they would get an analog voltage value that remains constant during the frame time.

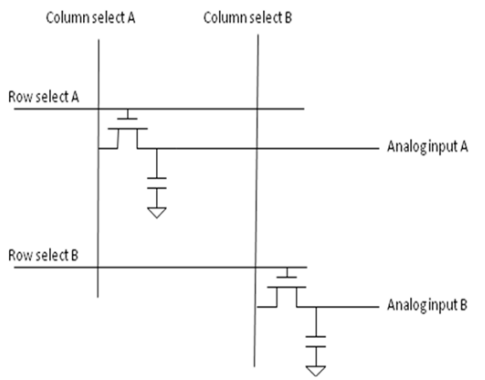

Figure 5: Schematic of an active matrix cell corresponding to one micromirror.

In order to perform an experimental verification of the working principle, we have fabricated micromirrors with SiGe used as the structural layer (See Figure 6). As a dummy CMOS stack, a 1000nm Si-oxide and $250 \mathrm{SiC}$ a 250 Sicxise is deposited. Both thin and thick electrode are pattened in one etch step where a resist mask is used for defining the thick electrode structures and the Si-oxide hardmask is used for defining the thin electrode structures. To create flat mirrors, a $1200 \mathrm{~nm}$ Si-oxide layer is deposited and planarised by ChemicalMechanical Polishing (CMP), stopping on the thick electrode. Next, a 400nm Si-oxide sacrificial layer is deposited and patterned to construct the anchors. Then the $330 \mathrm{~nm}$ optimized poly- SiGe structural layer is deposited by Chemical Vapor Deposition (CVD) at a chuck temperature of $460^{\circ} \mathrm{C}$ on top of a thin Ti/TiN adhesion layer. To reduce the roughness of this structural layer yet another CMP step is done. To obtain a higher reflectivity, a $5 \mathrm{~nm}$ barrier layer and a $30 \mathrm{~nm} \mathrm{Al} \mathrm{coating} \mathrm{is} \mathrm{sputtered} \mathrm{on} \mathrm{top} \mathrm{of} \mathrm{the} \mathrm{CMPed} \mathrm{SiGe.} \mathrm{At}$ last, a Si-oxide hardmask is deposited and then the $\mathrm{SiGe} / \mathrm{barrier} / \mathrm{Al} / \mathrm{Si}$-oxide stack is patterned before the mirrors are being released in HF Vapour.

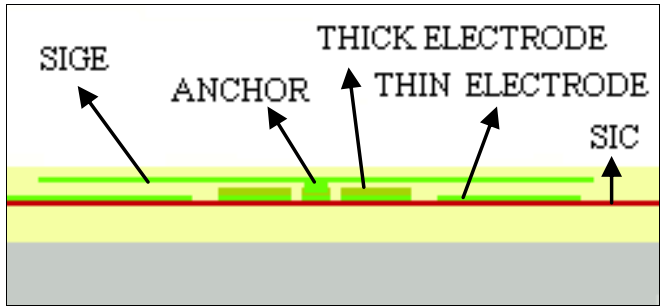

Figure 6: Schematic cross section of the SiGe micromirror process.
To verify if the fixed voltage on the inner electrode actually influences the pull-in voltage, a finite element simulation (COMSOL multiphysics) has been executed, considering two attracting electrodes at one side of the mirror as shown in Figure 1. The mirror size (length=width) and thickness are chosen to be $9.4 \mu \mathrm{m}$ and $0.35 \mu \mathrm{m}$ respectively.

In the first experiment the electrodes are equally high and wide as in Figure 3, which leads to a gap between the mirror and the electrodes of $0.5 \mu \mathrm{m}$. After every simulation cycle we increase the voltage of the outer "triangular" electrode and keep the same fixed voltage on the inner electrode. At some point the outer electrode's voltage is too high and the simulation does not reach a stable solution. This voltage corresponds to the pull-in voltage for that fixed voltage. In Figure 7 the pull-in voltage $V_{\text {pull-in }}$ is shown as a function of the fixed voltage $V_{\text {fixed }}$ on the inner electrode. Note that the curve decreases, but not sufficiently for our application.

In the second experiment we take the same mirror but with the electrode configuration of Figure 4. Since the gap between the fixed voltage electrode and mirror is smaller $(0.3 \mu \mathrm{m})$ than in the first experiment, this electrode has more influence on the mirror and likewise on the curve of the pull-in voltage. This set-up gives a good modulation of the pull-in voltage as a function of the fixed voltage value on the inner electrode.

As an experimental verification of the COMSOL simulation, a measurement was performed on the fabricated mirrors using a Laser Doppler Vibrometer (LDV). The mirror size and thickness of the experimentally tested design is respectively $20 \mu \mathrm{m}$ and $\sim 0.35 \mu \mathrm{m}$ (structural layer after CMP, including adhesion layer and reflective coating) and the gap between the mirror and the fixed electrode is $0.40 \mu \mathrm{m}$. The triangular waveform from Figure 8 was applied at the outer electrode at one side of the mirror. The $z$ displacement of a point at the mirror as a function of the fixed electrode voltage is shown in Figure 9. The same effect was observed: the fixed electrode voltage modulates the pull-in voltage and also the pull-out voltage (Figure 10). In the next paragraph, it will be demonstrated how variable pull-in can be applied for analog pulse width modulation of the mirror.

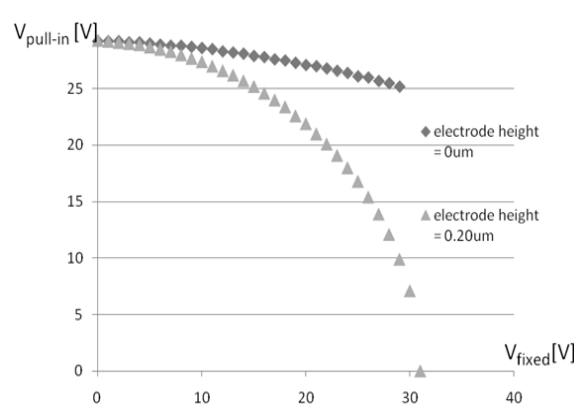

Figure 7. COMSOL simulation shows pull in voltage as a function of a fixed voltage.

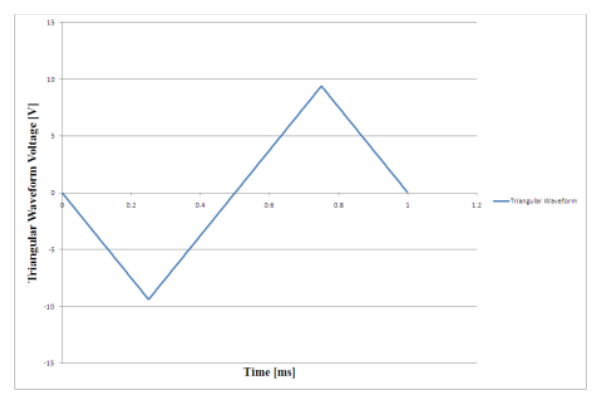

Figure 8. Triangular waveform applied to the outer electrode of the fabricated mirrors. 


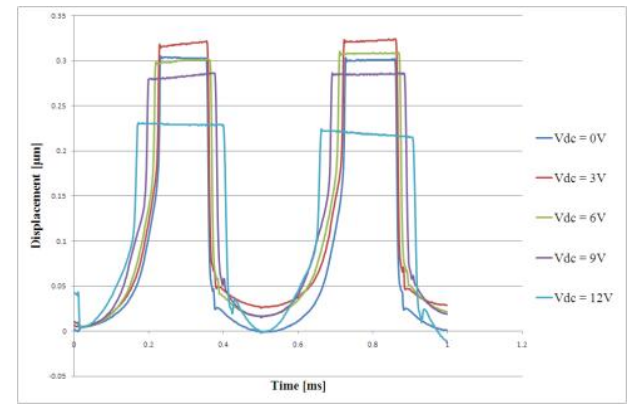

Figure 9. Displacement of the mirror as a function of the fixed electrode voltage, measured with LDV.

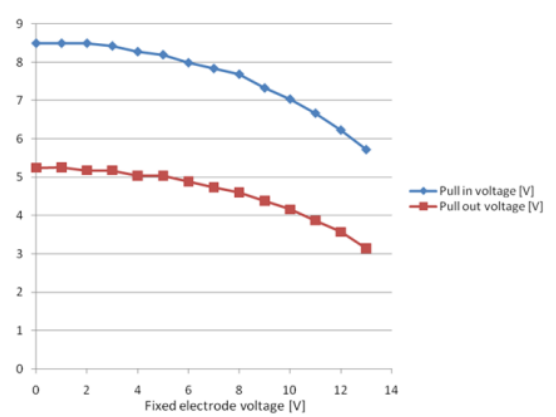

Figure 10. Measured pull-in and pull-out voltage as a function of a fixed voltage.
IV.

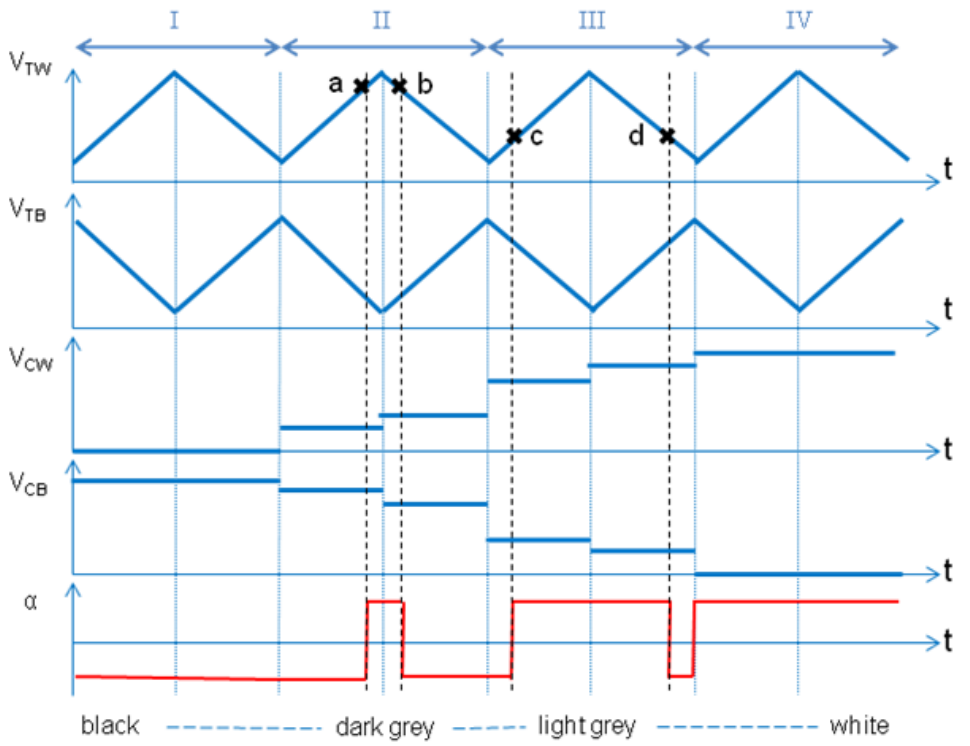

Figure 11: Theoretical control signals on the electrodes of the micromirror and mirror angle $\alpha$

In Figure 11 one can observe the theoretical control signals $\mathrm{V}_{\mathrm{TW}}, \mathrm{V}_{\mathrm{TB}}, \mathrm{V}_{\mathrm{CW}}, \mathrm{V}_{\mathrm{CB}}$ and the reaction of the mirror $\alpha$. The first signal $V_{T W}$ represents the triangular control voltage that is applied to an outer electrode.
When the mirror is pulled towards this electrode, the mirror reflects light through the lens. This corresponds to the "white" side. Signal $\mathrm{V}_{\text {ТB }}$ corresponds to the opposite outer electrode at the "black" side. The signal is a similar triangular curve, but in anti-phase. Note that these signals are chosen unipolar, but also bipolar triangular waveform could be applied. The two inner electrodes have signals $\mathrm{V}_{\mathrm{CW}}$ and $\mathrm{V}_{\mathrm{CB}}$, corresponding to the fixed voltage signals at respectively the white and black side. Signal $\alpha$ represents the deflection of the mirror compared to its resting state.

During period $\mathrm{I} \mathrm{V}_{\mathrm{CB}}$ is high and $\mathrm{V}_{\mathrm{CW}}$ is low. This way, the mirror is immediately attracted to the black side and stays there for the whole period. This corresponds to a duty cycle $\delta$ of $0 \%$ and results in a black pixel on the screen.

During the first half of period II, the constant voltage on the white side has increased and that on the black side has decreased. Because of this, at a certain point, the influence of $\mathrm{V}_{\mathrm{TW}}$ together with $\mathrm{V}_{\mathrm{CW}}$ becomes too strong, and makes the mirror flip to the other (white) side. To make the mirror flip at a chosen point a, we can for instance apply the constant voltage value $\mathrm{V}_{\mathrm{CW}}$ corresponding with a pull-in voltage at point a. Also we can apply the fixed voltage value $\mathrm{V}_{\mathrm{CB}}$ corresponding with the pull-out voltage at point a. This way pullin and pull-out work together at point a. During the second half, the pull-out voltage for point $b$ is applied a $\mathrm{V}_{\mathrm{CW}}$ and pull-in voltage for point $\mathrm{b}$ at $\mathrm{V}_{\mathrm{CB}}$. Note that the pull-out voltage is lower than the corresponding pull-in voltage. Here we get a duty cycle $\delta$ of $30 \%$ for instance, leading to a dark grey pixel.

Similarly in period III $\mathrm{V}_{\mathrm{CW}}$ is further increased, as $\mathrm{V}_{\mathrm{CB}}$ is decreased. Points $\mathrm{c}$ and $\mathrm{d}$ are achieved respectively earlier and later compared to the points $\mathrm{a}$ and $\mathrm{b}$ in period II. The duty cycle $\delta$ now is about $70 \%$, which leads to a light grey pixel.

During period IV $\mathrm{V}_{\mathrm{CW}}$ is set high and $\mathrm{V}_{\mathrm{CB}}$ low. The mirror is held at the white side for the whole period. $\mathrm{A}$ theoretical duty cycle $\delta$ of $100 \%$ is reached.

Choosing the right voltage values for $\mathrm{V}_{\mathrm{CW}}$ and $\mathrm{V}_{\mathrm{CB}}$ and the optimal triangular signals $\mathrm{V}_{\mathrm{Tw}}$ and $\mathrm{V}_{\mathrm{TB}}$, we can get an arbitrary deflection duty cycle of the mirror. This way of control implements PWM without needing an electronic comparator. Instead a 'comparator' is provided electromechanically through combination of constant and triangular signals.

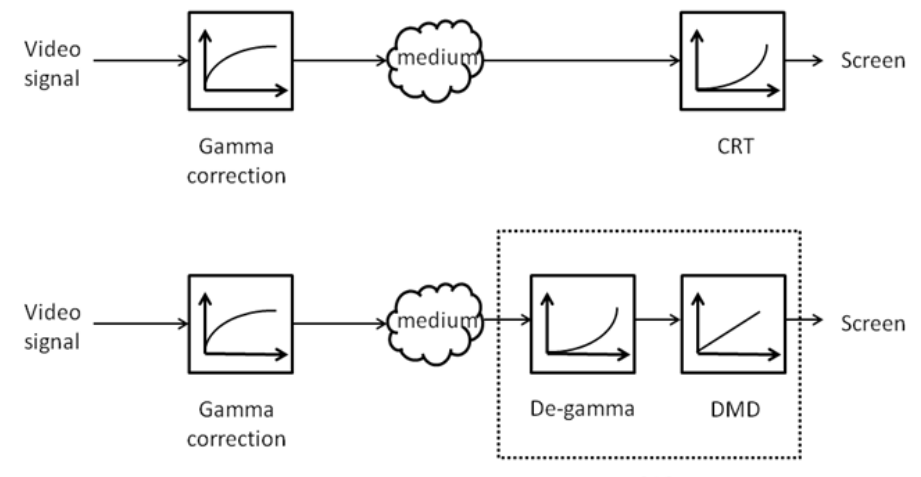

DLP

Figure 12: Schematical comparison between CRT and DMD. 
In the early days of television broadcast, video signals were gamma corrected to compensate the non-linear voltage-to-light characteristic of the CRT (see also Figure 12). This correction follows a logarithmic relationship, inverse to the CRT characteristic, which is a power-law relationship. Gamma corrected video signals are still common practice, so for example DMD needs the de-gamma process to 'decode' these video signals, in fact implementing the CRT non-linear characteristic, because DMD inherently has a linear voltage-to-light characteristic.

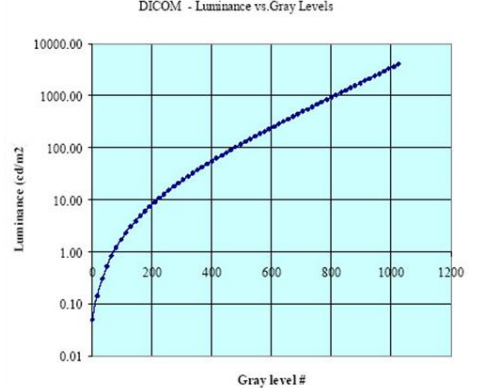

Figure 13. The DICOM curve - luminance versus grey levels (Source: [5]).

In Figure 13, we see an approximation of the lightness experienced through the human vision system as a function of the relative luminance observed. This is the DICOM standard used in medical displays [5]. This shows that dark levels can be better distinguished by the human eye than brighter levels. In Figure 14 a de-gamma curve is shown using a 7 bit linear output resolution. Because of the poor and equidistant output level distribution, the lower output levels lead to objectionable contours in the image [6] To overcome this contouring effect, a higher output bit depth is needed to get more levels at the low intensity side. In our design, we do not have to choose equidistant intensity levels and therefore there is no need to increase the output bit depth. We can choose the appropriate fixed voltage values in a way we have more dark output levels and less bright levels, meeting the psychometric lightness curve or the nonequidistant DICOM distribution.

Another feasible approach could be, adjusting the triangular waveforms into the 'gamma corrected' waveforms as illustrated in Figure 15, following the gamma response. By applying a linear distribution to the inner electrodes, the same result is obtained.

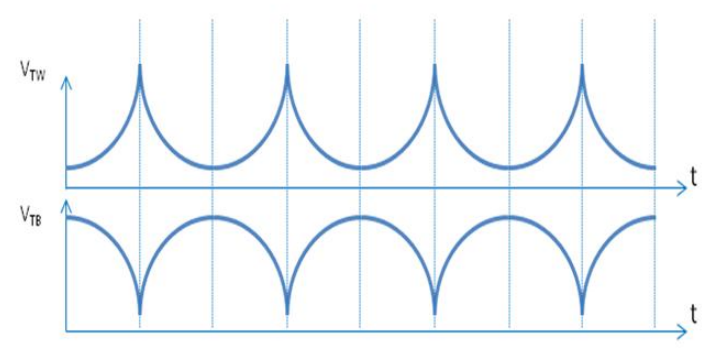

Figure 15: Gamma corrected 'triangular' waveforms

\section{CONCLUSION}

We have shown how PWM can be achieved by a micromirror without the use of a comparator in CMOS. This mirror design comprises four electrodes underneath. The inner two are chosen thicker than the outer two, implementing analog PWM by combining triangular and constant voltage signals. This results in less severe speed requirements for the on-chip electronics, reduced data rates, image processing hardware and memory compared to regular digital PWM. Electromechanical PWM also can implement the non-linear gamma grey scale distribution and overcome the contouring effect via appropriate control signals. An active matrix display with micromirrors has been presented with the use of only 2 transistors per mirror.

\section{ACKNOWLEDGEMENTS}

This work was performed in the framework of the IWT sponsored SBO project 'GEMINI' ('Generic Electronics and Microsystems INtegration Initiative'), IWT-nr 60046, 2007-2011, in collaboration with KULeuven and IMEC.

\section{REFERENCES}

[1] IWT-SBO project 'GEMINI' together with KULeuven and IMEC ('Generic Electronics and Microsystems INtegration Initiative'), IWT-nr 60046, 2007-2011.

[2] P. F. Van Kessel, L. J. Hornbeck, R. E. Meier, M. R. Douglass, “A MEMS-based projection display”, Proc. IEEE, Vol. 86, no. 8, pp. 1687-1704, 1998.

[3] O. Degani et al., "Pull-in study of an electrostatic torsion microactuator", Journal of micromechanical systems, Vol. 7, no. 4, 1998

[4] C.E. Tew, "Analog pulse width modulation cell for Digital Micromechanical Device", US 6,466,358 B2, 2002.

[5] A. Abileah, "DICOM Calibration for Medical Displays",

http://www.planar.com/Advantages/WhitePapers/docs/WP DICOM Calibration.pdf, 2005.

[6] V. Markandey et al., "Video processing for DLP display systems", Proc. SPIE, Vol. 2666, pp. 21-32, Image and Video Processing IV, Robert L. Stevenson; M. Ibrahim Sezan; Eds, 1996. 